\title{
Spatial Probabilistic Model of Block Failure Capacity of Piles in Clay
}

Indra Djati Sidi

Department of Civil Engineering, Institut Teknologi Bandung, Bandung 40132, Indonesia

\begin{abstract}
A probability based model of block failure capacity of pile foundation in clay soil under axial load is developed. The model was based on the first order second moment method. Instead of using point variability, the soil inherent variability is modelled as random field model. Based on this model, a reliability based factor of safety for designing pile group foundation, taking into account bock failure mechanism, is proposed. Furthermore, using simplified lognormal model, the relationship between the factor of safety used in design practice and target reliability may be derived explicitly.
\end{abstract}

Key words: Block failure, soil variability, random field, model error, reliability index.

\section{Introduction}

When a pile group is not very large, both theory and experience have shown that a pile group may fail as one unit by breaking into the ground before the load for each individual pile reaches its allowable design load. $[1,2]$. Sowers et al. [1] have shown that the minimum spacing to prevent group failure ranges from 1.75 diameters to 2.5 diameters, depending on the number of piles in the group. Nevertheless, block failure may be encountered for pile spacing at even 3 diameters to 6 diameters $[3,4]$, where the pile and the confined mass of soil work like a rigid unit. It is therefore necessary to investigate this group (or block) failure as an additional failure mode.

\section{Block Failure Formulation}

The ultimate bearing capacity $Q_{g u}$ of a pile group for the undrained condition is sufficiently modelled by superposition of the friction group capacity, $Q_{g f}$, and the base group capacity, $Q_{g b}$ (Fig. 1), as:

$$
Q_{g u}=Q_{g f}+Q_{g b}
$$

where,

$$
Q_{g f}=2 \int_{0}^{D_{f}} \int_{0}^{B} f S(x, y) \mathrm{d} x \mathrm{~d} y+
$$

Corresponding author: Indra Djati Sidi, Ph.D., associate professor, research fields: structure safety and reliability.

$$
2 \int_{0}^{D_{f}} \int_{0}^{L} f S(x, y) \mathrm{d} x \mathrm{~d} y
$$

and,

$$
Q_{g b}=\int_{0}^{B} \int_{0}^{L} q_{d}(x, y) \mathrm{d} x \mathrm{~d} y
$$

where, $D_{f}$ is the depth of foundation; $B$ is the width of group piles; $L$ is the length of pile group; $f_{s}(x, y)$ is the inherent variability of shear resistance of soil per unit area. For the case of homogeneous soil with negligible inherent spatial variability, i.e., $f_{s}(x, y)$ will reduce to $f_{s}$ and $q_{d}$, and Eq. (3) becomes:

$$
Q_{g u}=D_{f}(2 B+2 L) \cdot f_{s}+q_{d} B L
$$

The shear resistance $f_{s}$ may be assumed equal to undrained shear strength, and $q_{d}$ may be evaluated using the equation suggested by Terzaghi and Peck [2], that is

$$
q_{d}=1.2 c \cdot N_{c}+\gamma D_{f} N_{q}+0.4 \gamma B N_{\gamma}
$$

where, $N_{c}, N_{q}$ and $N_{\gamma}$ are Terzaghi bearing capacity factors; $c$ is the undrained shear strength of soil; $\gamma$ is the unit weight of soil. In the case of cohesive clay soil with angle of internal friction $\phi$ equal to zero, Skempton [5] has proposed the following simple expression for bearing capacity of a rectangular footing, as a function of soil shear strength and the dimension of the foundation itself: 


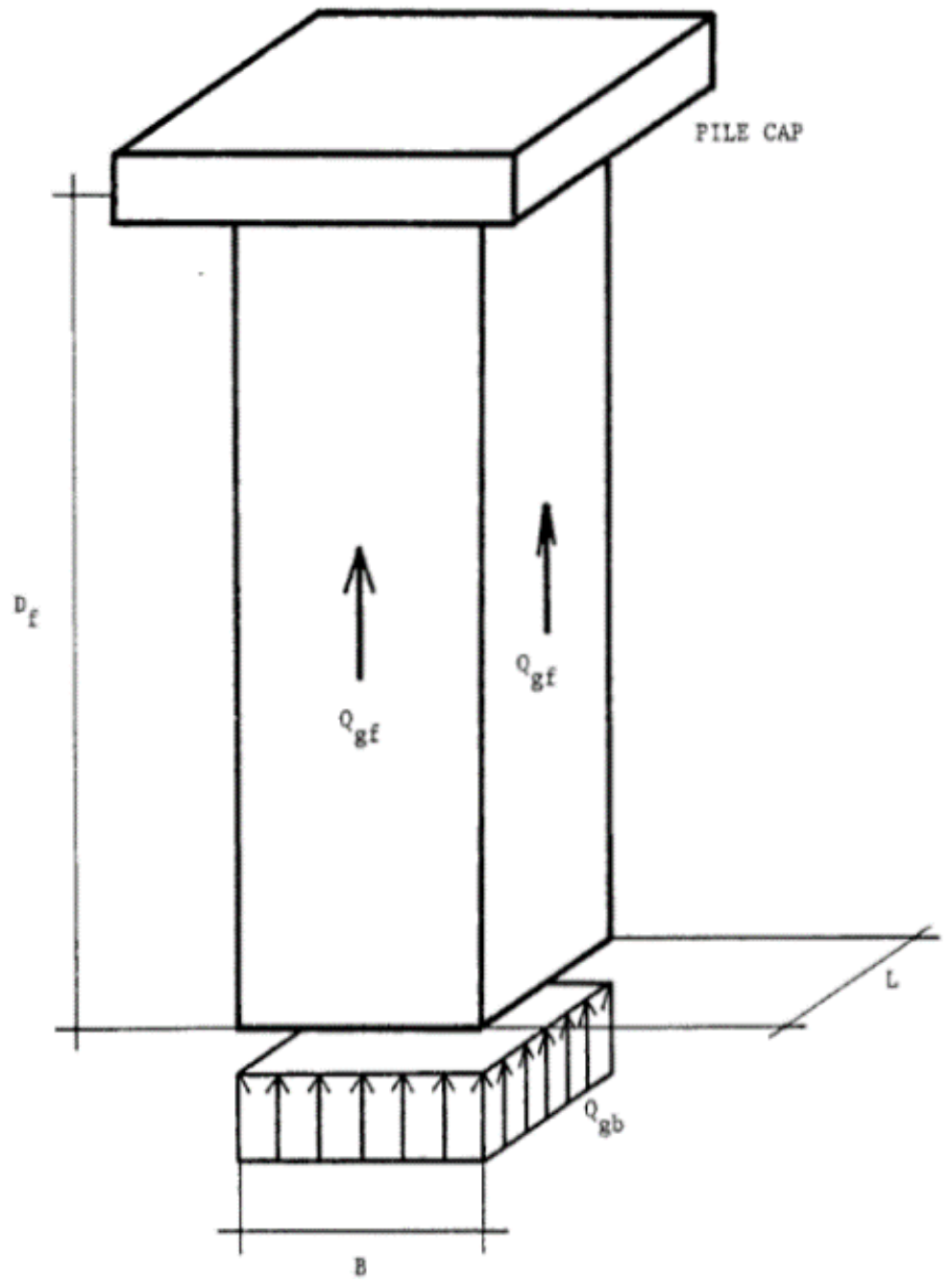

Fig. 1 Block ultimate capacity of a pile group.

$$
q_{d}=5 c\left(1+0.2 \frac{D_{f}}{B}\right)\left(1+0.2 \frac{B}{L}\right)
$$

Furthermore, he showed that the net base resistance $q_{d}$ becomes practically constant for values $D_{f} / B$ greater than 2.5, and may be taken equal to $9 c$. Both Eqs. (5) and (6) are widely used in the design practice.

\section{Probabilistic Model on Block Failure Mechanism}

Applying the first order analysis [6, 7] to Eqs. (2) and (3), the expected value of $Q_{g u}$ due to inherent spatial variability of the undrained shear strength may be expressed as [5]:

$$
E\left[Q_{g u}\right]=D_{f}[2 B+2 L] \cdot E\left[f_{s}\right]+B \cdot L \cdot E \cdot\left[q_{d}\right]
$$

Assuming that $Q_{g f}$ and $Q_{g b}$ are independent random variables, the variance of $Q_{g u}$ becomes:

$$
\operatorname{VAR}\left[Q_{g u}\right]=\operatorname{VAR}\left[Q_{g f}\right]+\operatorname{VAR}\left[Q_{g b}\right]
$$

where,

$$
\begin{gathered}
V A R\left[Q_{g f}\right]=4 V A R\left[\int_{0}^{\mathrm{D}_{\mathrm{f}}} \int_{0}^{B} f_{s}(x, y) \mathrm{d} x \mathrm{~d} y\right]+\angle \\
4 V A R\left[\int_{0}^{\mathrm{D}_{\mathrm{f}}} \int_{0}^{L} f_{s}(x, y) \mathrm{d} x \mathrm{~d} y\right]
\end{gathered}
$$

and,

$$
V A R\left[Q_{g b}\right]=V A R\left[\int_{0}^{\mathrm{B}} \int_{0}^{L} q_{d}(x, y) \mathrm{d} x \mathrm{~d} y\right]
$$

Consider the first term of Eq. (9), let

$$
A=\int_{0}^{D_{f}} \int_{0}^{B} f_{s}(x, y) \mathrm{d} x \mathrm{~d} y
$$


The variance of $A$ can be shown to be

$$
\begin{aligned}
& V A R[A]=\int_{0}^{D_{f}} \int_{0}^{D_{f}} \int_{0}^{B} \int_{0}^{B} \rho\left(\tau_{1}, \tau_{2}\right) \\
& V A R\left[f_{s}(x, y)\right] \mathrm{d} x_{1} \mathrm{~d} x_{2} \mathrm{~d} y_{1} \mathrm{~d} y_{2}
\end{aligned}
$$

where, $\tau_{1}$ and $\tau_{2}$ are spatial distances in directions $\mathrm{X}$ and $\mathrm{Y}$, respectively (Fig. 2 ) and $\rho\left(\tau_{1}, \tau_{2}\right)=$ correlation function of the undrained shear strength separated at distances $\tau_{1}$ and $\tau_{2}$ in $\mathrm{X}$ and $\mathrm{Y}$ axis, respectively [8].

A 2-dimensional variance function, $\Gamma_{2}\left(D_{f}, B\right)$, which relates the variance of spatial average within an area and the point variability may be defined for $f_{s}(x, y)$ as

$$
\begin{gathered}
\Gamma_{2}\left(D_{f}, B\right)= \\
\frac{\int_{0}^{D_{\mathrm{f}}} \int_{0}^{D_{f}} \int_{0}^{B} \int_{0}^{B} \rho\left(\tau_{1}, \tau_{2}\right) \mathrm{d} x_{1} \mathrm{~d} x_{2} \mathrm{~d} y_{1} \mathrm{~d} y_{2}}{D_{f}^{2} B^{2}}
\end{gathered}
$$

Hence, Eq. (12) becomes

$$
V A R[A]=D_{f}^{2} B^{2} \Gamma^{2}\left(D_{f}, B\right) V A R\left[f_{s}(x, y)\right]
$$

For the special case where the correlation structure is separable [9], the 2-dimensional correlation and variance function can be expressed in term of products of the respective one-dimensional function, namely,

$$
\rho\left(\tau_{1}, \tau_{2}\right)=\rho\left(\tau_{1}\right) \rho\left(\tau_{2}\right)
$$

and

$$
\Gamma^{2}\left(D_{f}, \mathrm{~B}\right)=\Gamma^{2}\left(D_{f}\right) \Gamma^{2}(B)
$$

allowing considerable mathematical simplification. Both triangular and double exponential correlation functions exhibit this "separable" property. Similarly, the second term of Eq. (9) and the variance of $Q_{g b}$ (Eq. (10)) can also be derived.

On the basis of procedures described in the aforementioned paragraphs, the mean and variance of pile block capacity due to inherent spatial variability of the undrained shear strength in a homogeneous soil deposit may be shown as

$$
E\left[Q_{g u}\right]=\left\{D_{f}[2 B+2 L)+\gamma_{b} B L\right\} \mu_{c}
$$

And

$$
\begin{gathered}
V A R\left[Q_{g u}\right]=\left\{4 B^{2} D_{f}^{2} \Gamma^{2}(B) \Gamma^{2}\left(D_{f}\right)+\right. \\
4 L^{2} D_{f}^{2} \Gamma^{2}(L) \Gamma^{2}\left(D_{f}\right)+ \\
\left.\gamma_{b}^{2} B^{2} L 2 \Gamma^{2}(B) \Gamma^{2}(L)\right\} \delta_{c}^{2} \mu_{c}^{2}
\end{gathered}
$$

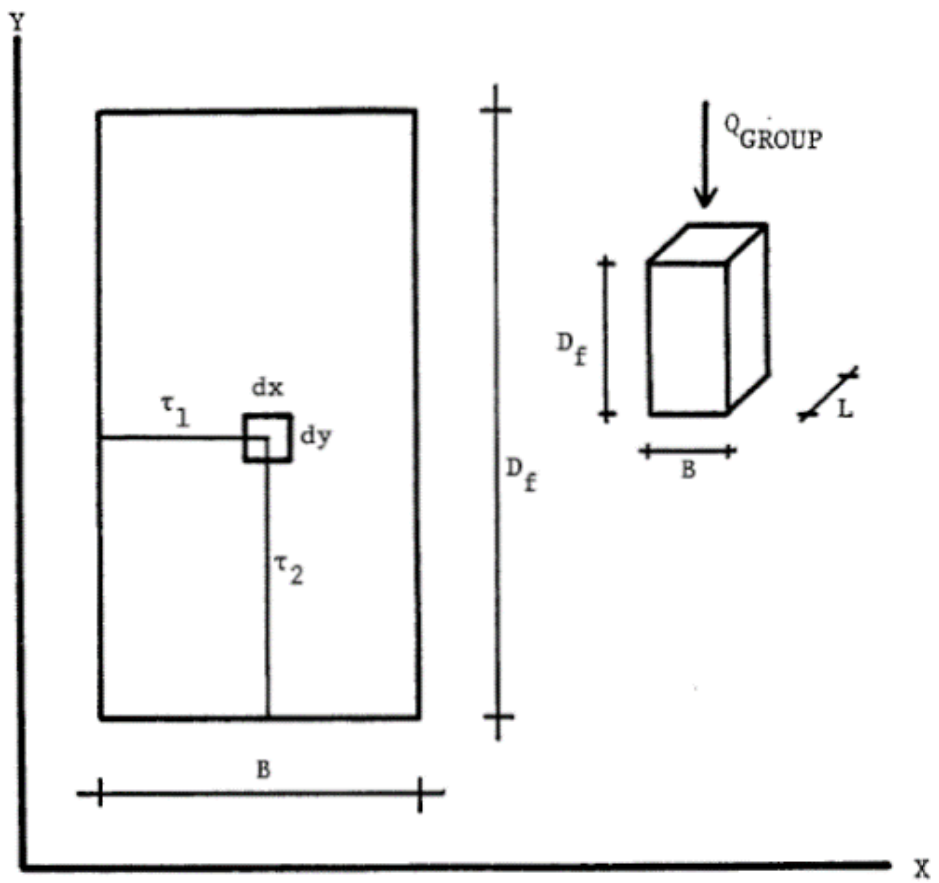

Fig. 2 Spatial distances in 2-dimensional random field. 
where, $\mu_{c}$ and $\delta_{c}$ are the mean and c.o.v of the undrained shear strength at a point representing the point variability of the soil shear sterngth, and $\gamma_{b}$ is defined as

$$
\gamma_{b}=\left(1+\frac{D_{f}}{B}\right)\left(1+\frac{B}{L}\right) S
$$

Eqs. (17) and (18) are derived based on the assumption that the correlation structure $\rho\left(\tau_{1}, \tau_{2}\right)$ of the undrained shear strength can be sufficiently represented by a separable type variance function (e.g., triangular or quadratic exponential type), and the contribution of the base to the block capacity is evaluated based on Skempton's equation given in Eq. (6). For the case typical of most pile foundation, where $B=L$ and $D_{f} / B \geq 2.5$, the c.o.v of $Q_{g u}$, is given by

$$
\begin{gathered}
\Omega_{Q_{g u}}= \\
\left\{\frac{8 \alpha_{f}^{2} \Gamma^{2}(B) \Gamma^{2}\left(D_{f}\right)+81 \Gamma^{2}(B) \Gamma^{2}(B)}{\left(9-4 \alpha_{f}\right)^{2}}\right\}^{0.5} \delta_{c}
\end{gathered}
$$

in which, $\alpha_{f}$ is the ratio of $D_{f}$ to $B$.

The effect of inherent spatial variability of the undrained shear strength $c$ on block failure mode has been evaluated as functions of the ratio of depth of penetration $\left(D_{f}\right)$ to the vertical scale of fluctuation of $c, \theta_{v}$, and $\alpha_{f}$ as presented in Fig. 3 for the typical cases of $B=L$ and $\alpha_{f} \geq 2.5$. The horizontal scale of fluctuation $\theta_{h}$ is much larger than the vertical scale of fluctuation $\theta_{v}$.

In this study, the ratio of $\theta_{h} / \theta_{v}$ equal to 9 [9] is used in addition to the c.o.v of 0.4 for $\delta_{c}$, similar to the case of a single pile, as the depth $D_{f}$ increases relative to $B$ and $\theta_{v}$, the averaging area increases, and hence the c.o.v of $Q_{g u}$ (in this case $\Omega_{Q_{g u}}$ ) decreases as shown in Fig. 3. Moreover, for a relatively short pile (e.g., $\alpha_{f}=$ 5), the percentage contribution of the base capacity $Q_{g b}$ to block capacity $Q_{g u}$ given by Eq. (1) is higher than those of a deep pile foundation (e.g., $\alpha_{f}=20$ ); this fact also contributes to the relatively higher $\Omega_{Q_{g u}}$ values at smaller $\alpha_{f}$ compared to those of higher $\alpha_{f}$. Fig. 3 shows that designing pile group using point variability data will lead to a very conservative design, as the variability of soil parameter would decrease in the case of pile foundation due the averaging effect.

\section{Model Error in Group Capacity}

Many assumption and simplification has been made

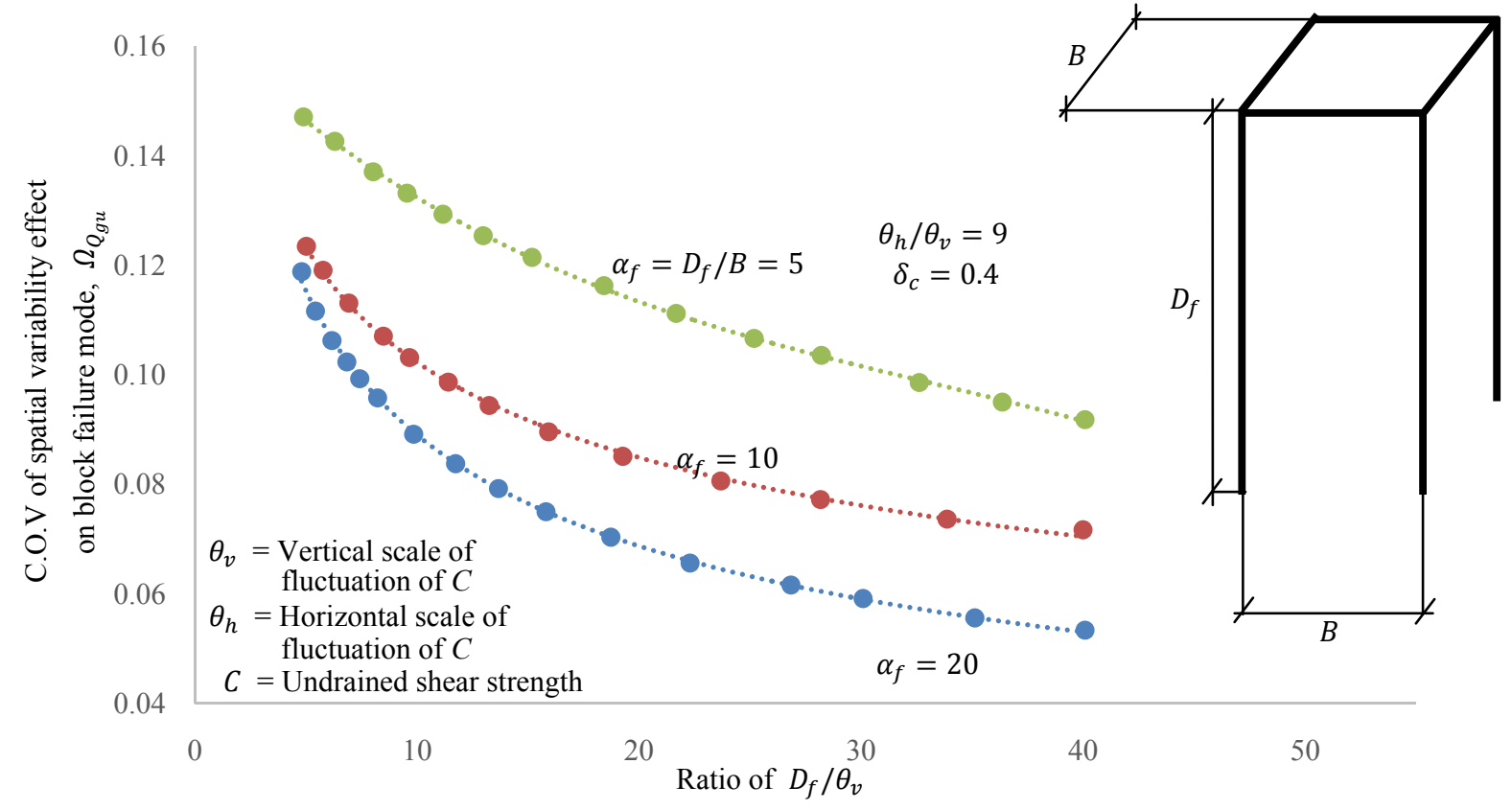

Fig. 3 The effect of spatial variability of undrained shear strength, $c$. 
in the formulation of block failure capacity of pile foundation due to the complexity of mechanic of soil response. The engineer purposely simplified the equation to guarantee a direct approach in designing a block of foundation and introduce a factor safety to account for imperfection. Hence, a difference between calculated and measured capacity cannot be avoided. Sidi [8] introduced random correction factor $N_{g}$ with mean value of one and coefficient variation of 0.06 may be used in the reliability formulation of block failure capacity to account for the imperfection. Taking into account the model error $\mathrm{N}_{\mathrm{g}}$, the true group capacity $Q_{g t}$ can then be written as:

$$
Q_{g t}=N_{g} \cdot\left[Q_{g f}+Q_{g b}\right]
$$

And the mean value of $\mathrm{Q}_{\mathrm{gt}}$ may be given by $E \cdot\left[Q_{g t}\right]=E \cdot\left[N_{g}\right] \cdot D_{f}[2 B+2 L] E \cdot\left[f_{s}\right]+B \cdot L \cdot E \cdot\left[q_{d}\right](22)$ And the coefficient variation of $Q_{g t}$ may be given by

$$
\Omega_{g t}=\sqrt{\Omega_{g u}^{2}+\Omega_{N_{g}}^{2}}
$$

where, $\Omega_{g u}=$ coefficient of $\mathrm{Q}_{\mathrm{gu}}$ given by Eq. (20), and
$\Omega_{g t}=$ the coefficient variation of the statistics of true capacity $Q_{g t}$ taking into account both the spatial inherent variability of soil parameter and the systematic model error of the block failure capacity, and may readily be used in the reliability formulation.

\section{Factor of Safety Based on Lognormal Model}

By assuming the load acting on the pile and the capacity block failure mode follow independent lognormal distribution, the safety index $\beta$ may be derived as

$$
\beta=\frac{\lambda_{g t}-\lambda_{L}}{\sqrt{\xi_{g t}^{2}+\xi_{L}^{2}}}
$$

where, $\lambda_{g t}$ and $\xi_{g t}$ are parameters of lognormal distribution of resistance $R$ whereas $\lambda_{L}$ and $\xi_{L}$ are parameters of lognormal distribution of resistance $L$, respectively. By introducing $\underline{\alpha}_{g t}$ as the ratio of nominal value of resistance used in design $\left(R_{n}\right)$ and the mean

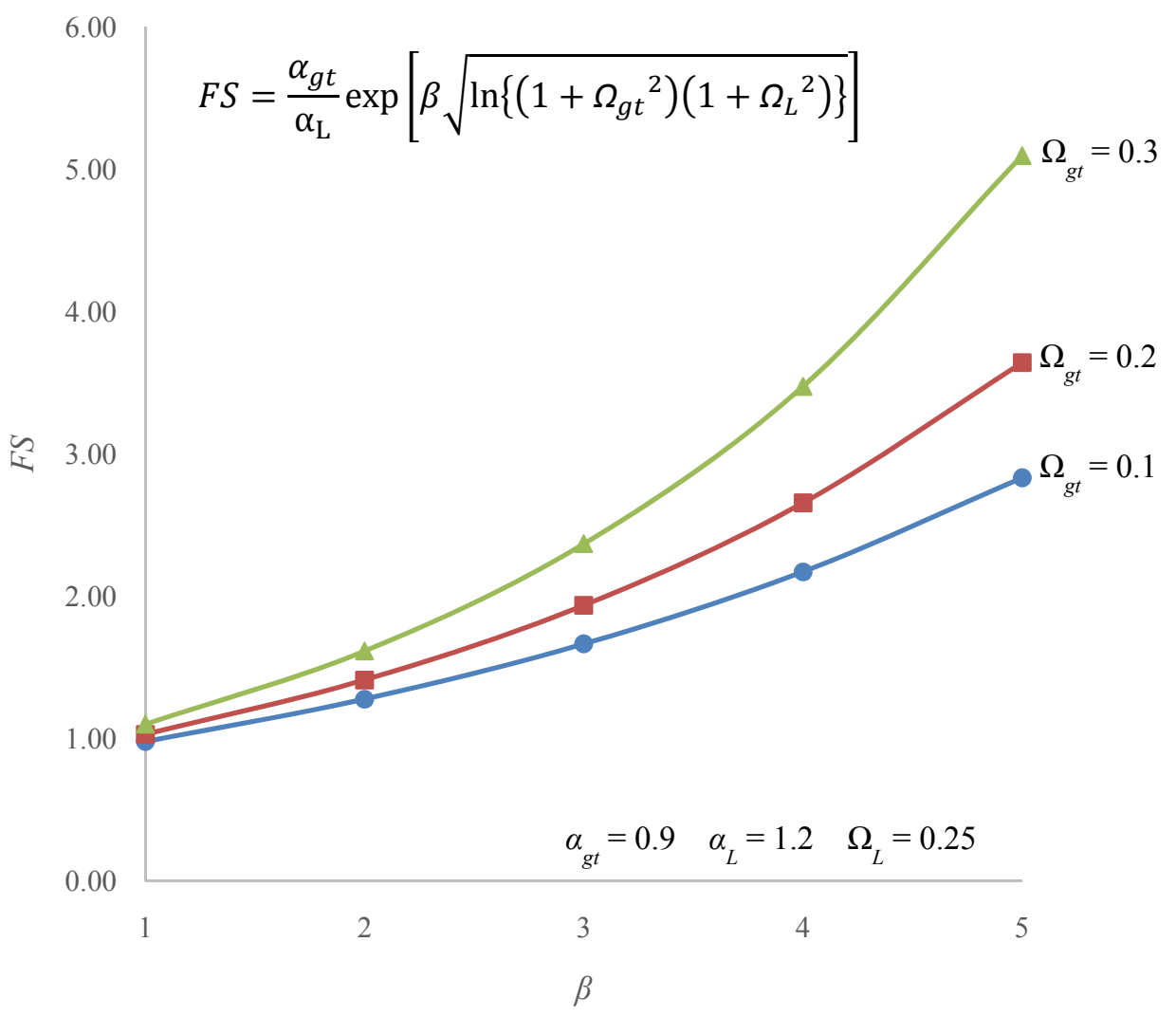

Fig. 4 Variation of factor of safety $F S$ with safety index $\beta$. 
value of R, $\mu_{g t}$, and $\underline{\alpha}_{L}$ as the ratio of nominal value of load used in design $\left(L_{n}\right)$ and the mean value of $L, \mu_{L}$, and by defining factor of safety $F S$ as the ratio of $R_{n}$ and $L_{n}$ given by:

$$
F S=\frac{R_{n}}{L_{n}}
$$

The factor of safety may be deriving as function safety index $\beta$ and the related coefficient variation of $R$ and $L$, as

$$
F S=\frac{\alpha_{g t}}{\alpha_{L}} \exp \left[\beta \sqrt{\ln \left(1+\Omega_{g t}^{2}\right)\left(1+\Omega_{L}^{2}\right)}\right]
$$

Eq. (26) shows that the factor of safety depends of the target reliability $\beta$ and the variation of $\mathrm{Q}_{\mathrm{gt}}$ and $L$ represented by its coefficient variation. The bigger the coefficient variation the bigger the factor of safety needed to achieve a certain targeted reliability. Of course if the designer is already taking conservative values in determining nominal design values of $Q_{g t n}$ and $L_{n}$ representing by $\alpha_{g t}$ of less than one and $\alpha_{L}$ factor of more than one, one will get a smaller $F S$ for a certain target reliability index $\beta$. Fig. 4 shows the variation of factor of safety with respect of targeted reliability index. The higher the coefficient variation of the block failure, the higher factor of safety needed for achieving a certain targeted reliability index $\beta$. Eq. (26) shows that the factor of safety is a function of both inherent variability of soil shear strength and systematic model error of block equation representing by $\Omega_{g t}$, and the variability of load given by its $\Omega_{L}$ which is readily to use for design purposes.

\section{Conclusions}

Based on a random field theory, the spatial variability of soil shear strength is modelled probabilistically taking into account horizontal and vertical correlation representing by its auto correlation function. Due to the averaging effect with respect of the area of block capacity, the point coefficient variation of inherent variability of soil shear strength would decrease significantly with the size (depth and width) of the foundation, and hence would lead to a smaller factor of safety needed to achieve a certain target of reliability index. By combining with the model error of block capacity, one could calculate the necessary traditional factor of safety needed to achieve a certain target reliability based on first order second moment method. The model takes into account variability of resistance governed by inherent variability, systematic model error, and the variability of the load itself. A simple lognormal reliability model has been introduced enabling one to determine the required factor of safety as a function of safety index or a certain acceptable risk, variability of soil, and load.

\section{References}

[1] Sowers, G. F., Martin, C. B., Wilson, L. L., and Fausold, M. 1961. "The Bearing Capacity of Friction Pile Groups in Homogeneous Clay from Model Studies." In Proceedings of 5th International Conference of Soil Mechanics and Foundation Engineering, 155-9.

[2] Terzaghi, K., and Peck, R. B. 1967. Soil Mechanics in Engineering Practice. New York: John Wiley.

[3] Peck, R. B., Hanson, W. E., and Thornburn, T. H. 1974. Foundation Engineering. 2nd ed. New York: John Wiley.

[4] Zeevaert, L. 1972. Foundation Engineering for Difficult Subsoil Conditions. New York: Van Nostrand.

[5] Skempton, A. W. 1951. "The Bearing Capacity of Clays.'In Proceedings of British Building Research Congress, 180-9.

[6] Ang, A. H-S., and Ellingwood, B. R. 1971. "Critical Analysis of Reliability Principles Relative to Design." In Proceedings of the 1 st International Conference Application of Statistics and Probability to Soil and Structural Engineering, 1755-69.

[7] Ang, A. H-S., and Tang, W. H. 1975. Probability Concepts in Engineering Planning and Design. Vol. I. New York: Wiley.

[8] Sidi, I. D. 1986. "Probabilistic Prediction of Friction Pile Capacities." Thesis of doctor of philosophy, Department of Civil Engineering, University of Illinois, Urbana.

[9] Vanmarcke, E. H. 1977. "Probabilistic Modeling of Soil Profiles," Journal of the Geotechnical Engineering Division 103 (GT11): 1227-46. 